\title{
Clinical diagnosis of fissure caries with conventional and laser-induced fluorescence techniques
}

\author{
C. H. Chu • E. C. M. Lo • D. S. H. You
}

Received: 13 September 2008 / Accepted: 5 February 2009/Published online: 4 March 2009

(C) Springer-Verlag London Limited 2009

\begin{abstract}
We studied the in vivo validity of dentinal fissure caries diagnosis by visual examination, bitewing radiography, and use of a laser-induced fluorescence device (DIAGNOdent). A total of 144 and second molars with macroscopically intact occlusal surfaces in 41 Chinese young adults were examined visually, by bitewing radiography, and by DIAGNOdent. Visual examination after pit and fissure opening was used as the reference standard. The sensitivity and specificity of detecting caries that had extended into the dentin were, respectively, 0.89 and 0.44 by visual detection of opacity or discoloration after air drying, 0.13 and 1.00 by bitewing radiography to detect radiolucency extending into the dentin, and 0.70 and 0.84 by DIAGNOdent testing with a cut-off score of 40 . Caries detection by a combination of visual examination and DIAGNOdent had a sensitivity of 0.67 and specificity of 0.94 . Receiver operating characteristic analysis showed that this combined approach was superior to the other methods.
\end{abstract}

Keywords Fissure caries · Diagnosis · Visual · Radiograph · DIAGNOdent

\section{Introduction}

There has been a general decline in the prevalence of dental caries owing to the increased use of fluoride in the form of fluoridated water, fluoride toothpaste, and fluoride agents that are applied professionally or at home. At the same

C. H. Chu $(\bowtie) \cdot$ E. C. M. Lo · D. S. H. You

The University of Hong Kong,

34 Hospital Road,

Hong Kong, SAR, China

e-mail: chchu@hku.hk time, the pattern of caries has also changed: the proportion of caries found in occlusal fissures has risen [4] and pit and fissure caries are now perceived as the predominant types [7]. The main reason for these changes is that fluoride inhibits enamel breakdown, so caries reaching the dentin tend to progress beneath a clinically intact enamel surface [7]. Given the difficulty in visually inspecting the fissures of molars, such cases of occlusal dentinal caries, known as "hidden caries", are commonly missed on visual examination and carious cavities are seen only at a late stage of disease.

It is important that hidden caries be diagnosed early so that appropriate preventive and restorative treatment can be provided promptly. Otherwise, it progresses to become unsightly, undermines the bulk of the dentin, and infects the pulp, making endodontic therapy the most likely option. One conventional diagnostic method relies on the radiolucency of hidden caries, which makes it detectable in a radiograph. Indeed, a clinical study of 200 young adults found that clinical evaluation with bitewing radiographic examination detected $106 \%$ more caries, mainly in the proximal surfaces, in the posterior permanent teeth than by clinical evaluation alone [8], which suggested that the detection rate of hidden caries depends on the prevalence of caries in the population and the frequency of bitewing radiographic examinations [24]. Although routine use of bitewing radiography could help identify teeth with hidden caries to allow early and conservative treatment, it is inaccurate in diagnosing occlusal enamel caries [19], and its detection rate for occlusal lesions is less than $50 \%$ of that for histological sectioning [30]. A re-evaluation of existing practices in caries detection and intensive research in developing new and more accurate detection methods are therefore needed.

The improvement of laser technology has permitted the use of laser-induced fluorescence (LF) for the early 
diagnosis of hidden occlusal caries [3]. DIAGNOdent (KaVo Dental, Biberachl Riss, Germany) is a small chairside battery-powered LF-based caries detection device that uses a quantitative optical method to detect mineral loss [18]. The tooth surface is illuminated by a red light (excitation wavelength at $655 \mathrm{~nm}$, modulated) that is produced by a laser diode and transmitted by an optical fiber. The laser induces fluorescence in mineralized tooth tissues, at a greater intensity in carious than in sound tooth tissues, which is then transmitted by an optical fiber to a photodiode with a long pass filter (wavelength $>680 \mathrm{~nm}$ ) in the detection device. The numerical value of the digital display (in units related to a calibration standard) correlates quantitatively with the intensity of the fluorescence detected and thus indicates the extent of caries. Lussi et al. [18] suggested that a score of 20 indicates caries extending into the dentin, and this reference has been used in other studies using DIAGNOdent [22, 28]. The DIAGNOdent device has been shown to have good in vitro precision, and this has been attributed to the good contrast between sound and carious regions when fluorescence is induced at red wavelengths and detected in near-infrared regions [26].

A recent systematic review concluded that a limiting aspect of studies investigating the performance of new methods to identify carious lesions is the difficulty in generalizing findings, especially in vitro ones, to the dental practice environment [5]. Hence, more evidence from clinical studies is needed to guide clinicians on the usefulness of new LF-based detection devices such as DIAGNOdent. For example, DIAGNOdent's reliability in detecting caries in primary teeth is reportedly high [29, 13], but its clinical performance has not been thoroughly or systematically examined on secondary teeth. The aim of this clinical study was to compare the in vivo validity of visual examination, bitewing radiography, and DIAGNOdent in diagnosing dentinal caries in permanent molars with early signs of fissure caries. As practiced by other researchers $[4,9,13]$, visual examination after pit and fissure opening with a fine carbide bur was used as the reference standard in this study.

\section{Materials and methods}

\section{Participant selection}

The participants of this clinical study were recruited from new students attending a free dental check-up offered by the University Health Services of the University of Hong Kong in August 2007. For each student, an initial visual examination, bitewing radiography, and LF measurement using DIAGNOdent were performed in the university campus dental clinic. Students were invited to participate if early signs of fissure caries were noted in one or more of their permanent molars and they agreed to have the carious molars treated after pit and fissure opening at a follow-up visit. The study was approved by the University Health Service of the University of Hong Kong. The study aim, procedures, safety, and benefits were explained to the students, and written consent was obtained before the study.

\section{Clinical examinations}

The initial visual examination was carried out by two dentists under optimal lighting with a front-surface dental mirror and a sickle-shaped explorer. The two dentists had been trained and calibrated in caries assessment in a pilot examination conducted before the study. The status of the participants' first and second molars was assessed and recorded after drying with compressed air using a 3-in-1 syringe. Plaque and food debris that obscured tooth surfaces were removed with compressed air and water through the 3-in-1 syringe and, if necessary, with the explorer, gauze, and cotton pellet. The target teeth were the first and second permanent molars that were macroscopically intact but with inconspicuous caries; hence, any students with teeth showing frank cavitation, obvious signs of enamel hypomineralization, or hypoplasia in the occlusal surface were excluded from the study. Students with teeth that had fillings, fissure sealant, restorations, or an orthodontic band were also excluded, as were those with non-vital teeth and teeth with apparent cavitation or caries in the mesial, distal, buccal, or palatal surfaces. The caries status of the fissures in the occlusal surfaces of the included molars was recorded according to codes (increasing in severity from V0 to V1, V3, V4) adapted from Ekstrand's visual scoring system [10], as shown in Table 1. A 10\% random sample was re-examined to monitor inter-examiner reproducibility.

After the visual examination, left and right bitewing radiographs were taken with Kodak Insight size 2 periapical FE-speed films (Eastman Kodak Co., Rochester, NY, USA) placed in a holder (Kwik Bite holder GDS1360; Kerr Hawe, Bradford,, UK) following a standard protocol. The X-ray machine (Gendex Oralix AC; KaVo Dental, Milan, Italy) was set at $65 \mathrm{kV}$ and $7.5 \mathrm{~mA}$, with an exposure time of $0.22 \mathrm{~s}$. Radiographs were developed by a standard automatic machine (Periomat; Durr Dental, Bietigheim, Germany) with fixer and developer from the machine's manufacturer. The image of each developed radiograph was captured with a digital camera (EOS 350D; Cannon, Tokyo, Japan) with a 60-mm lens and stored in JPEG format in a computer. Assessment of the radiographic image was performed on a 19-inch liquid crystal display (SP1908FP; Dell, Texas, USA) by an independent trained examiner who was not involved in the clinical examinations. The 
Table 1 Criteria used in visual examination, radiographic examination, and fissure opening

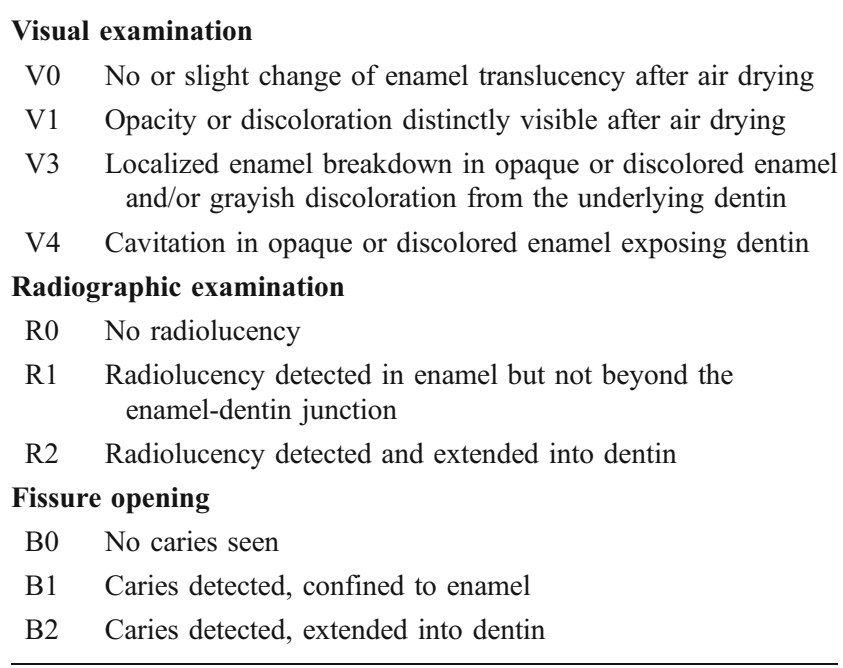

examiner looked for signs of dental caries in the occlusal surface of the molars and not in the proximal surfaces. Caries status scores (increasing in severity from R0 to R1, R2) were also adapted from Ekstrand et al. [10], as shown in Table 1 . A $10 \%$ random sample of the radiographs was re-examined at least 1 day later by the same examiner to assess intra-examiner reproducibility.

The status of the fissures of the included molars was then assessed with the LF detection device DIAGNOdent (KaVo Dental, Biberachl Riss, Germany) by a trained independent examiner who was not involved in the visual or radiographic examinations. The occlusal surface of the tooth was first cleaned with pumice and a rubber cup. The detection tip of the DIAGNOdent device was positioned on the target site and rotated around its long axis to record the highest value, with no time restriction. The highest reading shown on the display was recorded by a chairside assistant without informing the examiner of the reading. The procedure was repeated on a $10 \%$ random sample of teeth on the same day to assess the intra-examiner reliability. The DIAGNOdent device was calibrated twice a day following the procedures recommended by the manufacturer. A DIAGNOdent reading of 20 or higher signified dentinal caries [18].

Students were assumed to have early signs of fissure caries if any occlusal molar surface showed any one of the following criteria: a visual score of $\mathrm{V} 1, \mathrm{~V} 3$, or $\mathrm{V} 4$; a radiographic score of R1 or R2; or a DIAGNOdent score of 20 or higher (Table 1). These students were invited to return within 2 weeks for pit and fissure opening, followed by appropriate preventive and restorative treatment. For ethical reasons, students were excluded if they fulfilled all of the following three conditions: (1) no or only slight change in enamel translucency after air drying (V0); (2) no radiolucency detected in the radiograph (R0); and (3) a DIAGNOdent reading below 20 .

At the follow-up appointment, pit and fissure opening was performed by a trained independent dentist not involved in the previous examinations. The examiner carefully opened the fissures of the included molars with a fine carbide bur (Fissurotomy Micro NTF; SS White, Lakewood, NJ, USA) and looked for signs of caries, using codes (increasing in severity from B0 to B1, B2) adapted from Heinrich-Weltzien et al. [12], as shown in Table 1. Depending on the depth and extent of the cavity after careful caries removal, the examiner applied dental sealant, a preventive resin restorative, or a composite restorative to restore the tooth.

\section{Statistical analysis}

The data were analyzed with the software SPSS 15.0 for Windows (SPSS, Chicago IL, USA). Inter-examiner reproducibility of visual examinations and intra-examiner reliability of radiographic examinations were assessed using Cohen's Kappa statistics. Sensitivity and specificity in diagnosing dentinal caries using visual examination, radiographic examination, and DIAGNOdent according to appropriate cut-off scores were calculated from contingency tables. The Youden index, which is the value of [sensitivity+specificity - 1], was calculated for the three separate diagnostic methods and for combinations of different methods. Receiver operating characteristic (ROC) analysis was also performed to compare the diagnostic performance of the different methods of occlusal caries diagnosis. The cut-off level of significance was taken as $p=0.05$.

\section{Results}

A total of 1,178 new university students received a dental check-up and 135 (11.5\%) of them had signs of early caries in one or more of their molars. Forty-one $(30.4 \%)$ of the eligible students, 18 men and 23 women, agreed to attend a treatment appointment within 2 weeks after the screening examination, and were included in this study. The mean (SD) age of these 41 participants was 19.8 (1.6) years. The total number of molars showing possible early caries via visual examination was 144 . The reproducibility of the diagnostic assessments was very good: the Kappa coefficient of the inter-examiner agreement on the visual examination was 0.95 and total agreement was achieved in duplicate examinations of the radiographs. As for DIAGNOdent, $81 \%$ of duplicate readings had an absolute difference of no more than five units and the mean (SD) absolute difference was 3.2 (3.5). 
Upon initial visual inspection, $42(29.2 \%)$ of molars were status V0, 40 (27.8\%) were V1, 52 (36.1\%) were V3, and $10(6.9 \%)$ were V4. Nevertheless, pit and fissure opening was performed on all 144 teeth, because all DIAGNOdent scores were greater than 20 (range, 21-33). After fissurotomy, six (4.2\%) molars were found to be caries-free and $74(51.4 \%)$ had caries confined to the enamel, and these were restored with dental sealant. A further $64(44.4 \%)$ molars had caries extending into the dentin and were restored with resin or composite restorative (Table 2).

Only $12(8.3 \%)$ molars were positive for caries during radiography (Table 3). These teeth showed a radiolucent area in the enamel, either with or without dentin involvement, but all 12 were subsequently found to have caries extending into the dentin after pit and fissure opening. Bitewing radiography thus failed to distinguish clearly between fissure caries confined to the enamel and dentinal caries. In contrast, the mean DIAGNOdent score was significantly higher when the bitewing radiograph showed radiolucency in the dentin than when radiolucency was absent or confined to the enamel (Table 3).

The sensitivity and specificity of detecting fissure caries extending into the dentin varied according to different assessment methods and cut-off points (Table 4). Although a perfect specificity (1.00) was achievable via radiography with a diagnostic cut-off of R2 (radiolucency detected in the dentin), the sensitivity was very low $(0.13)$ and the Youden index was 0.13 . Visual examination with a V1 cutoff (opacity or discoloration distinctly visible after air drying) had a high sensitivity (0.89) but a rather low specificity (0.44) and a Youden index of 0.47 . If the diagnostic criterion was changed to V3 (localized enamel breakdown in opaque or discolored enamel and/or grayish discoloration from the underlying dentin), the sensitivity and specificity changed to 0.66 and 0.75 , respectively, with a Youden index of 0.41 .

Overall, molars with fissure caries extending into the dentin had a higher mean (SD) DIAGNOdent score than molars with only enamel caries ( $53 \pm 25$ vs. $26 \pm 5 ; p=0.003)$, but there was no significant difference in scores between molars with fissure caries confined to enamel and sound molars $(31 \pm 12$ vs. $26 \pm 5, p>0.05$ ). The cut-off value of 20 suggested by Lussi et al. [18] had a high sensitivity of 0.95 but a very low specificity of 0.11 in this study (Fig. 1). The specificity of diagnosing dentin caries using DIAGNOdent increased when the cut-off value was increased, but this was offset by a decline in sensitivity. The optimal cut-off value of 40 had a sensitivity and specificity of 0.70 and 0.84 , respectively, and the highest Youden index, of 0.54 . The area under the ROC curve was 0.79 .

When visual examination at V1 and DIAGNOdent were combined, the specificity of caries detection was generally higher than that of DIAGNOdent alone (Fig. 2). The area under the ROC curve also increased to 0.84 . At V1 and a DIAGNOdent cut-off score of 40 , a satisfactory level of sensitivity could be maintained (0.67), and the specificity was also good (0.94). The Youden index was the highest at these conditions, at 0.61 , compared with values ranging from 0.06 to 0.54 for visual examination, radiographic examination, or DIAGNOdent alone (Table 4).

\section{Discussion}

The detection of inconspicuous fissure caries on molars is often a challenge to clinicians, in particular when deciding whether surgical intervention should be performed. Although caries is generally not a life-threatening disease, operative treatments for late-stage disease involve the removal of tooth tissue and are irreversible. In contrast, carious lesions in early stages of disease can be remineralized or arrested by favorable changes in environmental factors such as the presence of fluoride ions and improvement in oral hygiene. An ideal diagnostic test for fissure caries therefore requires frequent recall to monitor caries progression and allow operative treatment at the right time, while being able to detect cases of disease with high sensitivity and exclude disease-free cases with high specificity in order to avoid overtreatment. This in vivo study evaluated the performance of visual examination, radiographic examination, and use of DIAGNOdent in the

Table 2 Status of fissure with visual examination and fissure opening $(N=144)$

Fissure opening

Visual examination

No or slight change of enamel translucency after air drying (V0)

Opacity or discoloration distinctly visible after air drying (V1)

Enamel breakdown and/or grayish discoloration from underlying dentin (V3)

Cavitation in opaque or discolored enamel exposing dentin (V4)

Total

\begin{tabular}{llll} 
Sound (B0) & Enamel caries (B1) & Dentin caries (B2) & Total \\
5 & 30 & 7 & 42 \\
1 & 24 & 15 & 40 \\
0 & 20 & 32 & 52 \\
0 & 0 & 10 & 10 \\
6 & 74 & 64 & 144 \\
\hline
\end{tabular}


Table 3 Status of fissure with radiographic examination and fissure opening $(N=144)$

\begin{tabular}{lllll}
\hline & Total & Fissure opening & & DIAGNOdent score \\
\hline Radiographic examination & & Sound (B0) & Enamel caries (B1) & Dentin caries (B2) \\
No radiolucency (R0) & 132 & 6 & 74 & 52 \\
Radiolucency in enamel (R1) & 4 & 0 & 0 & 4 \\
Radiolucency into dentin (R2) & 8 & 0 & 0 & $56(14)$ \\
\hline
\end{tabular}

*Bonferroni comparison: $\mathrm{R} 2>\mathrm{R} 1 \& \mathrm{R} 0(\mathrm{R} 2 \wedge \mathrm{R} 1, p=0.005 ; \mathrm{R} 2 \wedge \mathrm{R} 0, p<0.001 ; \mathrm{R} 1 \wedge \mathrm{R} 0, p=1.000)$

clinical detection of inconspicuous dentinal fissure caries of permanent molars in young Chinese adults. Our findings suggest that DIAGNOdent has a satisfactory validity, and that combined visual examination and DIAGNOdent is a superior method to others in fissure caries detection.

This study screened more than 1,000 students, of whom 41 had 144 molars showing possible inconspicuous fissure caries. The sample size is satisfactory when compared to those of related clinical studies [2, 9, 13]. Initial visual inspection was chosen as a screening method rather than tactile assessment with probing because the latter approach does not improve the validity of caries detection over visual examination [17]. In addition, probing pressure can damage the demineralized fissure and increase the risk for caries progression [10]. This study had a good inter-examiner agreement for visual examination, which could be attributed to the pilot examination and training before the main study. Other clinical studies have also reported good inter-examiner agreement using Ekstrand's caries index and examiner training $[2,22]$.

The low sensitivity and high specificity of visual examination at the V3 level (enamel breakdown) observed in our study agree with the findings of Attrill and Ashley [4]. When we changed the diagnostic level to detecting opacity and discoloration (V1), sensitivity improved but specificity diminished. The Youden index was also higher at the V1 level than at the V3 level, which agrees with the results of previous studies. Still, it is possible that examiners who knew the objectives of this study were likely to make an effort to examine the molars thoroughly, helped by optimal examination conditions, and thus concentrate on diagnosing hidden occlusal caries. This situation may be different from that experienced by general dental practitioners during a full routine clinical examination, when periodontal and oral mucosal conditions are assessed in addition to caries status. In practice, misdiagnoses of dentinal caries as enamel caries by visual inspection alone may be more likely and would allow a dentinal lesion to progress without notice.

Furthermore, radiographic examination was performed on a darker image than usual to improve diagnostic quality. Instead of using a conventional light box to assess images, this study used a 19-inch computer liquid crystal display to enlarge images for better visualization of radiolucency in dental hard tissue. The radiographs were interpreted with caution, bearing in mind the possibility of false-positive diagnoses that might arise from the Mach-band effect (an optical illusion of extra bands between sharp boundaries of light and dark areas) resulting in a tendency to see radiolucency in the dentin at the enamel-dentin junction of teeth with no dentinal caries. In the radiographic examination, the sensitivity was very low, as observed in previous studies $[4,11]$; however, the specificity of diagnosing early or small carious lesions in the dentin was very high. Hence,
Table 4 Sensitivity and specificity of diagnosing fissure caries extending into dentin using different methods and cut-off points $(N=144)$

*Youden Index=A (Sensitivity) $+\mathrm{B}$ (Specificity) -1

\begin{tabular}{llll}
\hline Diagnostic methods & Sensitivity (A) & Specificity (B) & Youden Index* \\
\hline Visual examination (V1) & 0.89 & 0.44 & 0.47 \\
Visual examination (V3) & 0.66 & 0.75 & 0.41 \\
Radiographic examination (R2) & 0.13 & 1.00 & 0.13 \\
DIAGNOdent (20) & 0.95 & 0.11 & 0.06 \\
DIAGNOdent (35) & 0.75 & 0.71 & 0.46 \\
DIAGNOdent (40) & 0.70 & 0.84 & 0.54 \\
DIAGNOdent (45) & 0.56 & 0.89 & 0.45 \\
Visual examination (V1) + DIAGNOdent (35) & 0.72 & 0.86 & 0.58 \\
Visual examination (V1) + DIAGNOdent (40) & 0.67 & 0.94 & 0.61 \\
Visual examination (V1) + DIAGNOdent (45) & 0.55 & 0.94 & 0.49 \\
\hline
\end{tabular}


Fig. 1 Sensitivity and specificity of caries detection according to DIAGNOdent cut-off value used

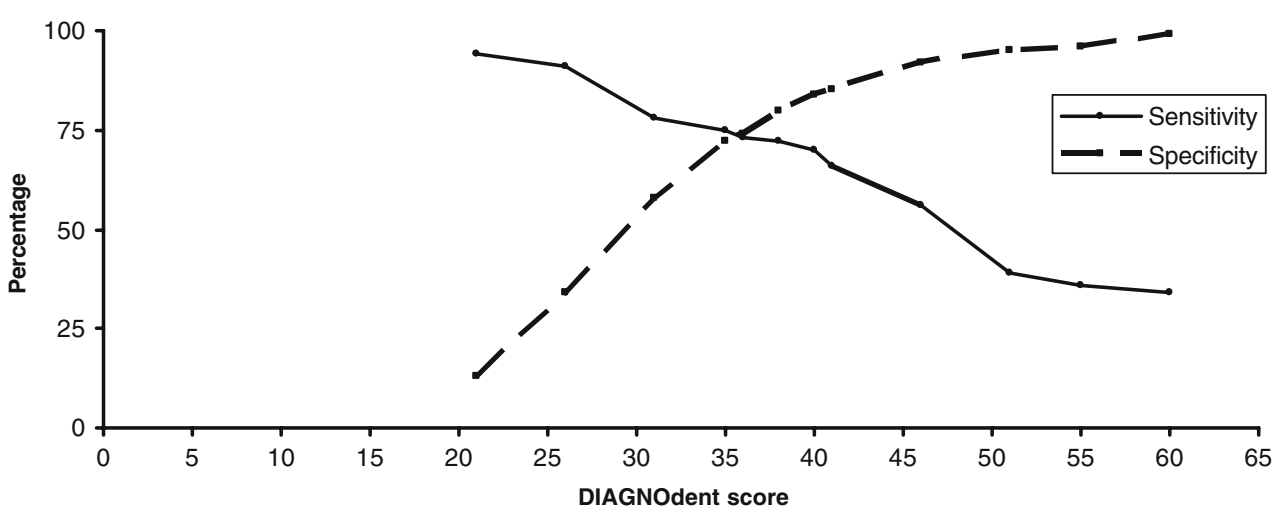

despite the technique's low sensitivity, dentists in their clinical examinations should always look for occlusal caries in radiographs. This recommendation has also been suggested by Poorterman et al. [21], who found areas of radiolucency beneath half of the sealed fissures of 17-year-olds and beneath a quarter of those of 20-year-olds in a Dutch population. Finally, this study aimed to evaluate conventional rather than digital radiography. Future investigations are needed to test the validity of digital radiography in fissure caries detection, especially with $\mathrm{CCD} / \mathrm{CMOS}$ sensors, which would offer good contrast and spatial resolution.

This study found inter-examiner agreement to be satisfactory, according to the absolute difference of DIAGNOdent score - that is, more than $80 \%$ of the assessments had an absolute difference in DIAGNOdent score of not more than 5 . Compared with visual examination, the use of DIAGNOdent required less rigorous training in achieving a good agreement in caries assessment. Kappa statistics for interexaminer agreement with DIAGNOdent assessment in previous clinical studies have been shown to be less than satisfactory [2, 25]. It has also been noted that caries detection in vivo yields higher DIAGNOdent scores than in vitro [22], especially in pigmented sites [22]. The median DIAGNOdent score in a stained fissure can be 5 to 7 units higher than in a fissure that is opaque or not discolored at a similar fissure depth [11]. Stains in fissures are unlikely to be removed by pumice or a rubber cup before DIAGNOdent assessment. In the laboratory, intrinsic and extrinsic stains can be removed by proteolytic solution such as hypochlorite [20], but this method is not commonly practiced in clinical settings. Furthermore, deep fissures [15] and the presence of calculus and organic debris [14] will also increase the DIAGNOdent score, thereby increasing the rate of falsepositives in caries assessment. Despite these shortcomings, we obtained acceptable sensitivity and satisfactory specificity values for DIAGNOdent, and our results are comparable to those of other studies of both permanent and primary teeth $[4,18]$.

Bader and Shugars [6] found that DIAGNOdent demonstrated greater sensitivity but poorer specificity than visual examination when they reviewed 25 studies, and Ricketts [23] concluded that DIAGNOdent is associated with a greater probability of false-positive diagnoses than visual examination. In contrast, more recent in vivo and in vitro studies have reported that DIAGNOdent has good specificity $[22,13,25]$. As adopted by other researchers [4, $18,22]$, a value of 20 was used as our initial cut-off point for performing pit and fissure opening. However, the diagnostic criterion for a positive test result among young adults in fluoridated areas should be stricter to give a lower
Fig. 2 Sensitivity and specificity of caries detection according to visual examination plus DIAGNOdent cut-off value used

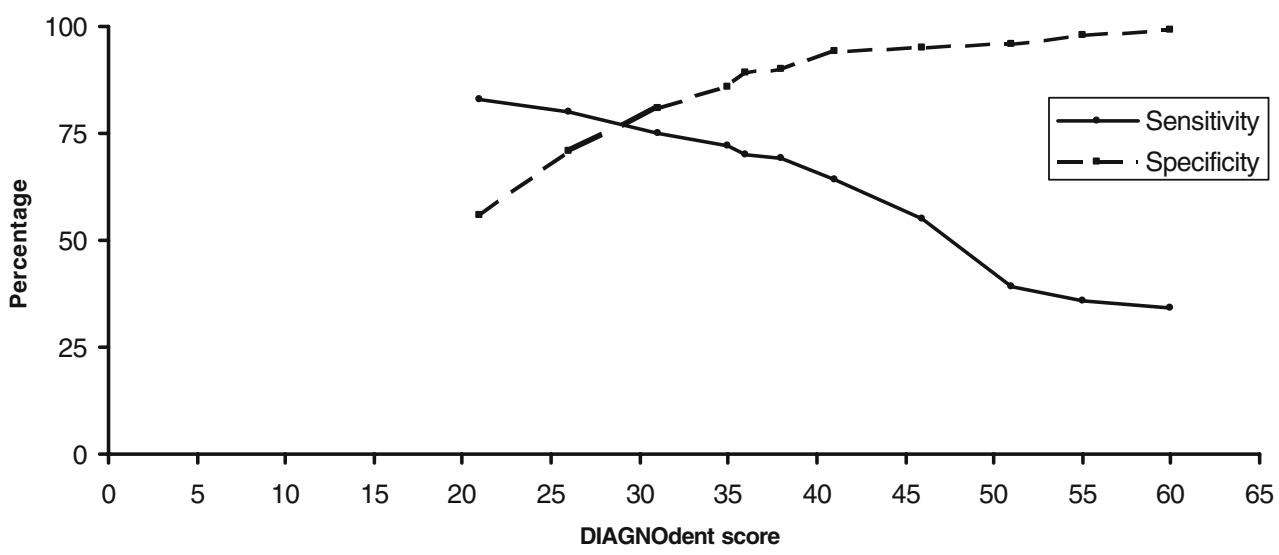


false-positive rate because the caries' prevalence is expected to be already low. In their clinical study, Kavvadia and Lagouvardos [13] used a higher cut-off point than is usually recommended for detecting caries in dentin. Likewise, the results of this study showed that the optimal cut-off point should be higher than 20 , so as to identify more true negatives (non-dentin caries) and achieve good specificity. We found that DIAGNOdent with a cut-off value of 40 has a sensitivity of 0.70 and specificity of 0.84 .

The findings of our ROC analysis are also similar to those of other clinical studies [22, 25]. Some researchers consider ROC analysis to reflect diagnostic performance more comprehensively than sensitivity and specificity, because it indicates overall validity of the assessment. Nevertheless, because this single summary value does not capture alternative options for diagnostic threshold (i.e., the cut-off score for diagnosing occlusal dentin caries), ROC analysis does not have a direct application in clinical diagnostic situations, and clinicians still need to select an appropriate threshold from the many points in the ROC curve. For this reason, some clinicians may prefer to keep using sensitivity and specificity in their comparisons of different diagnostic methods. The ideal caries diagnostic test has sensitivity and specificity values of 1 and an area under the ROC curve of 1, but these parameters never reach 1 in real life and some trade-offs must be made [7]. Youden [30] proposed an index to evaluate the appropriateness of a diagnostic test. The Youden index required for a test to be considered useful is typically more than 0.6 , but Baelum et al. [7] concluded that no caries diagnostic test has consistently been shown to fulfill this requirement. This study also found that no diagnostic test or combination of tests fulfilled this requirement, except the combination of visual examination and DIAGNOdent. The combined method of visual examination with the V1 criterion and DIAGNOdent with a cut-off score of 40 had a Youden index of 0.61 , and was therefore a superior method of fissure caries detection in this study.

A limitation of this study is that it did not record the lesion depth. Indeed, the reliability and calibration of carious lesion depth is very difficult to perform. DIAGNOdent is more reliable in detecting the presence of dentin caries (qualitative capacity) than the depth of the lesion (quantitative capacity) [1]. Porphyrins, which are synthesized by microorganisms in caries lesions, are the organic molecules mainly responsible for caries fluorescence during DIAGNOdent use [16]. Hence, there is actually a poor correlation between DIAGNOdent score and mineral content [27]. Another limitation is that there is no easy or objective way to decide whether a caries diagnosis is correct or not [7]. Pit and fissure opening by the use of a fine carbide bur was considered the reference standard in this and other studies $[4,9,13]$. However, sound teeth cannot be routinely evaluated in the same way for ethical reasons, because early enamel caries that could be remineralized would be unnecessarily removed.

\section{Conclusions}

The results from this in vivo clinical study provide valuable information on detecting inconspicuous fissure caries in young adults in Hong Kong, and probably in other optimally fluoridated areas, using visual examination, radiographic examination, DIAGNOdent, or a combination of examination methods. We conclude that visual examination observing opacity or discoloration after air drying in young adults is a sensitive tool in caries detection, but its specificity is low. Film-based bitewing radiography has the reverse performance characteristics. The LF-based DIAGNOdent device we tested has an acceptable sensitivity and specificity. Caries detection based on the combined findings from visual examination and DIAGNOdent offers a good level of specificity and a reasonable level of sensitivity, and can be considered an acceptable method for diagnosing fissure caries involving dentin.

Acknowledgements This study was supported by the University of Hong Kong and was not funded by a commercial company related to the manufacturer of DIAGNOdent. The authors would like to thank Dr. Trevor Lane for editing the manuscript.

\section{References}

1. Astvaldsdóttir A, Holbrook WP, Tranaeus S (2004) Consistency of DIAGNOdent instruments for clinical assessment of fissure caries. Acta Odontol Scand 62:193-198. doi:10.1080/00016350410001612

2. Angnes V, Angnes G, Batisttella M, Grande RH, Loguercio AD, Reis A (2005) Clinical effectiveness of laser fluorescence, visual inspection and radiography in the detection of occlusal caries. Caries Res 39:490-495. doi:10.1159/000088185

3. Akarsu S, Köprülü H (2006) In vivo comparison of the efficacy of DIAGNOdent by visual inspection and radiographic diagnostic techniques in the diagnosis of occlusal caries. J Clin Dent 17:53-58

4. Attrill DC, Ashley PF (2001) Occlusal caries detection in primary teeth: a comparison of DIAGNOdent with conventional methods. Br Dent J 190:440-443

5. Bader JD, Shugars DA, Bonito AJ (2002) A systematic review of the performance of methods for identifying carious lesions. J Public Health Dent 62:201-213. doi:10.1111/j.1752-7325.2002. tb03446.x

6. Bader JD, Shugars DA (2004) A systematic review of the performance of a laser fluorescence device for detecting caries. J Am Dent Assoc 135:1413-1426

7. Baelum V, Nyvad B, Gröndahl HG, Fejerskov O (2008) The foundations of good diagnostic practice. In: Fejerskov O, Kidd E (eds) Dental caries. The disease and its clinical management, 2nd edn. Blackwell Munksgaard, Oxford, pp 104-118

8. Chu CH, Chung BT, Lo EC (2008) Caries assessment by clinical examination with or without radiographs of young Chinese adults. Int Dent J 58:265-268 
9. Costa AM, Bezzerra AC, Fuks AB (2007) Assessment of the accuracy of visual examination, bite-wing radiographs and DIAGNOdent on the diagnosis of occlusal caries. Eur Arch Paediatr Dent 8:118-122

10. Ekstrand KR, Ricketts DN, Kidd EA (1997) Reproducibility and accuracy of three methods for assessment of demineralization depth of the occlusal surface: an in vitro examination. Caries Res 31:224-231

11. Francescut P, Lussi A (2003) Correlation between fissure discoloration, DIAGNOdent measurements, and caries depth: an in vitro study. Pediatr Dent 25:559-564

12. Heinrich-Weltzien R, Weerheijm KL, Kühnisch J, Oehme T, Stösser L (2002) Clinical evaluation of visual, radiographic, and laser fluorescence methods for detection of occlusal caries. ASDC J Dent Child 69:127-132

13. Kavvadia K, Lagouvardos P (2008) Clinical performance of a diode laser fluorescence device for the detection of occlusal caries in primary teeth. Int J Paediatr Dent 18:197-204. doi:10.1111/ j.1365-263X.2007.00913.x

14. Krause F, Braun A, Frentzen M (2003) The possibility of detecting subgingival calculus by laser-fluorescence in vitro. Lasers Med Sci 18:32-35. doi:10.1007/s10103-002-0241-7

15. Lundberg P, Morhed-Hultvall ML, Twetman S (2007) Mutans streptococci colonization and longitudinal caries detection with laser fluorescence in fissures of newly erupted 1st permanent molars. Acta Odontol Scand 65:189-193. doi:10.1080/00016350701302516

16. Lussi A, Hibst R, Paulus R (2004) DIAGNOdent: an optical method for caries detection. J Dent Res 83 Spec No C:C80-83

17. Lussi A, Francescut P (2003) Performance of conventional and new methods for the detection of occlusal caries in deciduous teeth. Caries Res 37:2-7. doi:10.1159/000068226

18. Lussi A, Megert B, Longbottom C, Reich E, Francescut P (2001) Clinical performance of a laser fluorescence device for detection of occlusal caries lesions. Eur J Oral Sci 109:14-19. doi:10.1034/ j.1600-0722.2001.109001014.x

19. Mejàre I, Kidd EAM (2008) Radiography for caries diagnosis. In: Fejerskov O, Kidd E (eds) Dental caries. The disease and its clinical management, 2nd edn. Blackwell Munksgaard, Oxford, p 76
20. Mendes FM, Pinheiro SL, Bengtson AL (2004) Effect of alteration in organic material of the occlusal caries on DIAGNOdent readings. Braz Oral Res 18:141-144. doi:10.1590/S180683242004000200009

21. Poorterman JH, Weerheijm KL, Groen HJ, Kalsbeek H (2000) Clinical and radiographic judgement of occlusal caries in adolescents. Eur J Oral Sci 108:93-98. doi:10.1034/j.16000722.2000.00791.x

22. Reis A, Mendes FM, Angnes V, Angnes G, Grande RH, Loguercio AD (2006) Performance of methods of occlusal caries detection in permanent teeth under clinical and laboratory conditions. J Dent 34:89-96. doi:10.1016/j.jdent.2005.04.002

23. Ricketts D (2005) The eyes have it. How good is DIAGNOdent at detecting caries? Evid Based Dent 6:64-65. doi:10.1038/sj. ebd.6400346

24. Ricketts D, Kidd E, Weerheijm K, de Soet H (1997) Hidden caries: what is it? Does it exist? Does it matter? Int Dent J 47:259265

25. Rodrigues JA, Hug I, Diniz MB, Lussi A (2008) Performance of fluorescence methods, radiographic examination and ICDAS II on occlusal surfaces in vitro. Caries Res 42:297-304. doi:10.1159/ 000148162

26. Shi XQ, Welander U, Angmar-Månsson B (2000) Occlusal caries detection with KaVo DIAGNOdent and radiography: an in vitro comparison. Caries Res 34:151-158. doi:10.1159/000016583

27. Shi XQ, Tranaeus S, Angmar-Månsson B (2001) Validation of DIAGNOdent for quantification of smooth-surface caries: an in vitro study. Acta Odontol Scand 59:74-78. doi:10.1080/ 000163501750157153

28. Silva BB, Severo NB, Maltz M (2007) Validity of diode laser to monitor carious lesions in pits and fissures. J Dent 35:679-682. doi:10.1016/j.jdent.2007.05.005

29. Wenzel A, Fejerskov O (1992) Validity of diagnosis of questionable caries lesions in occlusal surfaces of extracted third molars. Caries Res 26:188-194

30. Youden WJ (1950) An index for rating diagnostic tests. Cancer 3:32-35. doi:10.1002/1097-0142(1950)3:1<32::AIDCNCR2820030106>3.0.CO;2-3 